\title{
Entretien avec Yves Bonnefoy sur la traduction poétique (été 1994)
}

\author{
Sergio Villani \\ (Université York)
}

omment en etes-vous venu a vous intéresser a la traduction, en particulier a la traduction litteraire?

A la traduction littéraire seule, encore que je me serais volontiers laissé retenir - dans une autre vie - par des projets de traduction de textes philosophiques et les problèmes qu'ils posent: constat, alors, et surtout, de l'impossibilité de traduire: car les concepts, qui constituent pour l'essentiel ce mode-là du discours, sont moins des universaux que le produit de structures propres à chaque langue. Ils ne prennent pas la réalité par le même bout, même quand on veut croire que c'est le cas. Freedom, ainsi, n'est pas notre liberté, speech ne soupçonne pas toutes les acceptions et tous les emplois de parole, 11 y a de toute notion d'une langue à la plus proche notion dans la langue la plus voisine un important décalage, et quand on en vient à vouloir traduire de façon tant soit peu sérieuse, ce qui apparait aussitôt, ou devrait le faire, c'est qu'il faudrait avant toutes choses reprendre les concepts à leur origine, puis dans leur histoire en chacune des deux langues qui sont en jeu. C'est seulement au terme de ce travail, nécessairement comparatif, c'est à son terme s'il en est un, que l'on pourrait traduire sans perpétuer de malentendus. Traduire une pensée pour ce qu'elle fut et non la rêver dans la sienne propre.

En vérité, et je dis cela sans goût pour le paradoxe, le texte poétique, qui aura été mon grand soucl et l'objet de toutes mes traductions, est certainement plus facile à traduire que le texte philosophique, pour ne rien dire de la banale page de prose très quotidienne où les idiotismes de l'intellect sont à leur plus foisonnant. Car dans le poème, remarquez-le, que trouve-t-on, qui orientent, décident, sinon de grands symboles qui, étant nés d'observations faites au rebord entre parole et silence, entre notre savoir des choses et leur arrière-plan d'impénétré - et c'est alors de l'universel -, sont, eux, communs à bien des nations, en Occident en tous cas? Quand Yeats regarde s'envoler, revenir, crier les cygnes sauvages de Coole Park; quand il les retrouve, maintes années plus tard, apparemment les mêmes, apparemment toujours jeunes à l'horizon de sa vie qui passe, nous n'avons pas besoin, pour comprendre, de quoi que ce soit de plus que la désignation du cri rauque, de l'aile qui se déplie, du vacarme de l'eau, des "great broken rings" de l'essor des cygnes dans le ciel: c'est comme si la poésie effaçait ces réseaux de concepts qui n'auraient parlé de la vie, du temps, du destin qu'au prix d'abstractions jamais élaborées de même façon dans les langues de Berkeley, de Hegel ou de Malebranche. La pensée voue les langues à la solitude, ce fut là la tragédie de l'Europe; mais la poésie les rapproche. Et si le poème s'offre mieux que la prose à la traduction, eh bien, ajouterai-je, la grande poésie, pour sa part, le fait infiniment plus, à son tour, que la médiocre, elle est ce qu'il y a de plus facile à traduire, 
puisque c'est elle qui s'est le mieux établie dans l'économie, commune à tout être humain, du rapport de l'existence à son lieu terrestre. - Demeurent, certes, je ne l'oublie pas, j'en fais constamment l'expérience, quelques obstacles, disons, sur la belle voie qui semble libre. Car les concepts se maintiennent dans le travail des symboles, dans l'intuition que sont les images. Relativisés par la visée poétique, exposés par elle à une intimité de l'objet que par vocation ils refusent, ils ne s'obstinent pas moins, c'est au point qu'il faudrait peut-être partir des poèmes, où lls sont ainsı̀ à l'épreuve, pour en commencer la critique, celle d'où se dégageraient plus en profondeur les points de passage entre penser en anglais, penser en français, ou avoir pensé en grec, en araméen.

Et bien passionnante serait-elle, cette recherche qui puiserait dans le passé poétique de quol défiger l'affrontement des systèmes conceptuels qui sous-tendent les idiomes: puisque l'histoire de l'Occident, ce ne fut en somme que l'effet de ces côtoiements aveugles, de ces malentendus, de ce malentendre, sur les esprits, et cela depuis l'origine, avec nos contresens dans la lecture de Platon, nos emplois abusifs d'Aristote, pour ne rien dire de cette Bible des Septante où apparait, si je ne me trompe, le mot agapé: une de ces approximations qui peuvent être porteuses de vérité, d'encouragement à l'espérance. - Toutefois, malgré quelques expériences épisodiques, tentations plutôt que travaux, ce n'est pas sur ce plan que je me sens responsable. Et en revanche, et sans doute parce que j'ai voulu de toujours participer à cette recherche que l'on nomme la poésie, j'ai voulu, de toujours aussi, m'exercer à la traduction littéraire et formuler des remarques à son propos. En quatrième, au lycée, j'avais commencé - quelques pages, sans plus, mais avec un intérêt que je ne trouvais pas à simplement la lecture de ce récit - de traduire Le livre de la jungle, que nous avions au programme. C'était sans doute parce que je ne savais pas assez d'anglais pour m'attacher à ce texte de par l'intérieur de ses mots, de par leur pratique du monde, il me fallait donc lui rendre un peu de substance avec d'autres mots, ceux-ci familiers, et aussi peu appropriés ces nouveaux vocables fussent-lls, je le crains, à ce que Kipling avait en esprit. Et là se marque ce qui est, à mon sens, une des faiblesses possibles de toute entreprise de traduction: à un texte qui est un corps vivant, respirant du fait de toutes les connivences qui peuvent s'établir entre des mots d'un même parler, consanguins, on substitue ce qui, obligé à leur trame, ne peut plus se faire qu'une marqueterie de notions, sans racines mêlées sous la surface des phrases.

Néanmoins j'ai recommencé, et deux ou trois ans plus tard j'al voulu absolument traduire un de ces classiques que l'on apercevait au lycée (je n'avais pas d'autres occasions d'en connaitre): The Rime of the Ancient Mariner. Cette fois je fis la traduction tout entière, et peut-être avais-je raison de m'y obstiner, car j'étais très profondément remué par des vers comme

They were the first that ever burst Into that silent sea,

et 11 m'est aujourd'hul évident que ces allitérations mystérieuses ou pour mieux dire augurales me désignaient un niveau second, dans l'invisible, de la perception, de l'expérience de l'être, et m'incitaient donc à tenter de vivre de cette façon, poétique, les mots de ma propre langue. Il ne s'agissait plus, cette fois, de substituer 
aux connotations des concepts anglais, celles dans les vocables français, qui ne déferaient pas trop l'organicité du texte, il s'agissait de recommencer dans mes mots à moi le dépassement de la saisie ordinaire que Coleridge opérait si puissamment dans "the first" ou, et surtout, "silent sea." Il s'agissait moins de rendre en français un sens que d'entrer en poésie.

J'al traduit The Rime, détestant d'ailleurs le mot "ballade" que je ne savais pas éviter - puisque "le dit," ce n'est pas meilleur - mais je n'al pas gardé ce travail qui n'avait rien pour que je l'estime; et je m'avise que j'ai passé bien du temps ensuite avant que je ne me remette à traduire. Sans doute al-je fait, en 1950, et publié peu après, la traduction d'une brève pièce de Leonora Carrington que je trouvais, et trouve toujours, très belle, très émouvante. Mals ce ne fut pas avant 1955 que je me mis sérieusement à traduire; et, au moins en surface, ce fut beaucoup par hasard. Il se trouvait que je m'étais lié d'amitié avec Pierre Jean Jouve, l'année d'avant; et que Pierre Leyris, que Jouve connaissait bien, commençait alors la préparation de sa grande édition collective d'un Shakespeare français. Jouve suggéra à Leyris de me proposer une des plèces, ce qu'il fit, il m'offrit Jules César. J'acceptal, et pour répondre à son voeu de quelques pages d'essai, je traduisis la première scène de l'oeuvre avec un enthousiasme qui signifiait, d'évidence, un désir longtemps refoulé. Désir de traduire? Désir peut-être plutôt, à ce moment de ma vie, de me mesurer à la langue anglaise, à sa poésie dont je percevais les grands moyens spécifiques, bien différents de ceux de la poésie française mais susceptibles aussi de nous aider à chercher d'autres voies en nous, porteurs de mots français, héritiers d'une prosodie: de nous aider à mieux vivre. Je me suis expliqué sur ce point, il y a longtemps désormais. Il me semble qu'une des grandes fonctions de la traduction est de mettre en évidence, de dénoncer, les limites dans lesquelles s'enferment, par paresse à vivre, les traditions poétiques dont le traducteur a hérité d'une et qu'il a à subir, par conséquent, quand il se tente poète comme il le faut quand on traduit un poème.

\section{I m'a semble que vous faisiez une distinction entre traduire et transposer. Comment entendez-vous ces deux termes?}

Je n'en retiendral qu'un, et qui est traduire. Transposer, c'est un mot que j'ai employé une fois, dans un essai très ancien, et même dans son titre, déjà, mais c'était entre guillemets et pour référer à une proposition qu'un traducteur d'Hamlet avait faite et dont j'entendais me démarquer. Que pourrait-ce être que "transposer," quand il s'agit de la poésie? Garder d'une oeuvre quelques éléments signifiants, pour les greffer à une autre souche? Oui, c'est permis mais dans ce cas, et si cela vaut, ce n'est plus Saxo Grammaticus mais Shakespeare, ce n'est plus Euripide mais Racine: autant dire qu'on a oublié le texte de l'origine, et avec lui le voeu de rencontre, voire de communion entre deux cultures, qui est dans le projet de traduire. Il n'y a pas deux façons de faire, sur les confins de deux langues, seule vaut celle qui se propose de tout comprendre, de tout restituer de loeuvre considêrée, ou en tout cas de n'en sacrifier que ce que l'auteur aurait consenti d'abandonner pour sa part, si son expérience n'eût qu'à ce prix pu ne pas se dénaturer. Et pour cette fidélité au plus haut niveau tous les moyens, certes, sont bons, y compris ceux qui peuvent sembler l'infidélité la plus criante: mais c'est alors qu'on a constaté, justement, qu'en sacrifiant cet inessentiel on allait sauver 
ce qui importe.

Infidélité, et criante? Ce sera par exemple au plan des rythmes, quand on se permettra, comme je crols qu'on le peut et même le doit, d'abandonner le parti prosodique du texte original au profit d'un qui est né du possible que l'on porte en sol, et qui est contraint, évidemment, par le passé de la langue au sein de laquelle la traduction va se faire. Car on peut préserver, d'une langue à une autre, des symboles; comprendre les sentiments d'un poète et les revivre avec des mots étrangers à ce qu'ils furent; mais comment pourrait-on, en français, langue sans accents bien marqués, préserver les mètres et rythmes d'une langue où il y a des temps forts dans chaque mot, et donc des pieds dans les vers, non simplement des syllabes: ce quil induit une prosodie profondément dissemblable et à nos vers réguliers, d'ailleurs aujourd'hui caducs, et à nos vers libres? Il faut accepter cet écart, ou d'autres, d'entrée de jeu, comme l'accession à la liberté quí va nous permettre de nous approcher de l'oeuvre à traduire avec toute l'ampleur de nos moyens, dont aucun ne sera de trop; et sachant, aussi bien, que l'on ne réagit au fait poétique, en loccurrence cette oeuvre, que si on laisse monter du profond de soi des rythmes qui ne peuvent être que ceux du corps que l'on est, des expériences qu'on a vécues. Traduire n'est pas singer! Questionnement d'une liberté, c'est le droit, c'est même le devoir d'être soi-même tout aussi libre, et avec bonne conscience.

\section{Faut-il etre poete pour traduire la poesie?}

Évidement, mais tout le monde l'est en puissance, il ne s'agit que de le vouloir assez sérieusement et assez longtemps pour que cette sorte de conscience du monde, et de la vie, s'anime, se mette en place. Et l'oeuvre que l'on veut traduire, poésie comme elle est, est justement ce qui va inciter à cette recherche de soi: après quoi, s'il faut attendre vingt ans pour y parvenir, eh bien, pourquoi ne pas attendre vingt ans? De toutes façons, et ce n'est pas là me contredire, une conscience poétique pleine, durable, c'est une limite inaccessible. Auteur et traducteur n'auront fait, sur cette voie ardue, que chercher en même terrain, à leur distance à chacun - et qui les fait parfois se rapprocher, parfois aussi se perdre de vue de la crête interdite, dans ses nuêes.

\section{Mais peut-on traduire une oeurre dont on ne peut pas revivre les situations emotionnelles?}

Sans doute pas, et c'est pourquoi il ne faut songer à traduire que les poètes que l'on aime vraiment beaucoup, ce qui signifie qu'on les comprend, qu'on peut revivre leurs sentiments et leurs expériences, sinon réellement, dans sa propre vie, du moins de façon imaginative. Sans cet attachement instinctif, il n'y aurait que lectures en surface, où le contresens fleurirait parmi des images flétries. Mais c'est entre une personne et une autre qu'il y a proximité ou éloignement, ce n'est pas entre deux époques, et je ne crois guère qu'on soit aujourd'hui incapable de reparcourir les voles que prenait l'affectivité chez Homère ou Virgile, ou Dante ou Shakespeare, du moins quand c'est chez eux le projet de la poésie qui prend le pas sur leurs rapports à la société et à ses problèmes. Il y a des sentiments, des façons d'être qui nous sont inintelligibles, et je dirais même antipathiques, dans la Divine 
Comédie. Mais c'est alors parce que Dante est encore en decà de son ambition spécifiquement poétique, et nous le comprenons à nouveau quand nous savons voir que ces adhésions à des valeurs, à des attitudes que nous avons éventées il en est d'autres aujourd'hui pour nous prendre à semblables pièges - ne sont que sa timidité, sa limite: tandis que s'il laisse à son émotion un cours plus libre, Paolo, Francesca paraissent, ou bien c'est l'extraordinaire chant XXVII du Purgatotre, nous nous sentons remués au plus profond de notre être et comme révélés à nous-mêmes, malgré les siècles. Prenons un autre exemple, dans les Sonnets de Shakespeare. Sommes-nous incapables, au XXe siècle, de partager l'inquiétude de celui qui conjure là le beau jeune homme de procreer, parce que sinon 11 mourrait sans avoir rendu à la nature son dû? Nous ne croyons plus à la grande chaîne de l'Etre, c'est vrai, dans laquelle chaque personne est un maillon tôt rompu, ce qui exige un remplacement qu'il faut donc prévoir, préparer, sinon c'est le péché même. Mais nous ressentons toujours ce qu'il y a de suprêmement réel dans l'instant, si court, où un être a intacte en lui la beauté, l'éclat des jeunes années: or c'est cette fugacité, ce qui agite Shakespeare, c'est ce qui lui fait désirer que l'enfance naisse de la jeunesse, si bien que nous le comprenons, tout de même, nous le comprenons instinctivement, comme dans le cas des premières mesures de la Symphonie pastorale.

Dans ce cas de Shakespeare, faut-il sacrifier les formes archaiques de son anglais, qui contribuent considérablement au caractère de sa poessie?

Je ne crois pas que les aspects pour nous archaïques du texte de Shakespeare aient le moindre rapport à ce qui en fait de la poésie. Ces aspects, Shakespeare lui-même ne les percevait évidemment pas comme tels, il écrivait avec les moyens d'une langue parfaltement vivante, il est allé avec ces moyens jusqu'au bout de son expérience, et c'est celle-ci seule qui compte, non le vieillissement de ses mots. Mais vous me direz peut-être que vous pensiez à des vocables, à des formes syntaxiques qui engagealent sur des chemins que nous n'avons plus, vers la cime qui nous importe? Eh bien, si tel est le cas, nous en prendrons d'autres, de ces chemins: passant des anciens aux nôtres aussi résolument - bien que, certes, non sans regrets - que nous renonçons, bien forcés, quand nous traduisons "Eo Romam" dans Virgile, à l'impression d'immédiateté que les Latins recevaient de ce qu'on nomme en syntaxe l'accusatif de la question quo. Nous devons, c'est là le premier axiome de toute théorie de la traduction, traduire dans notre langue, en son état rigoureusement présent, qui seul nous permet de penser avec tout nous-mêmes à ce qu'un texte nous offre; et évoquer dans nos traductions françaises contemporaines ce que Shakespeare a d'archaïque pour son lecteur anglais d'aujourd'hui. non: ce serait l'éconduire d'un présent de la poésie où il a droit de parler autant que tout autre parmi nous. 